\title{
Energy-Efficient Computation Offloading for Wearable Devices and Smartphones in Mobile Cloud Computing
}

\author{
Claudio Ragona, Fabrizio Granelli \\ University of Trento, Italy \\ claudio.ragona@studenti.unitn.it,granelli@disi.unitn.it
}

\author{
Claudio Fiandrino, Dzmitry Kliazovich, Pascal Bouvry \\ University of Luxembourg, Luxembourg \\ \{name.surname\}@uni.lu
}

\begin{abstract}
Wearable devices are becoming increasingly popular and are expected to become essential in our everyday life. Despite continuous improvement of hardware, the lifetime of mobile devices and their capabilities still remain a concern. Small size of batteries of smart watches, glasses, helmets and gloves limits the amount of computing, storage and communication resources. Mobile cloud computing can augment the capabilities of wearable devices by helping to execute some of the computing tasks in the cloud. Such computational offloading helps to preserve battery power at the cost of more intensive communications with the cloud. In this paper, we present a model and comprehensive analysis for computational offloading between wearable devices and clouds in realistic setups.
\end{abstract}

\section{INTRODUCTION}

Wearable devices are expected to become essential for our day life in the nearest future. Smart watches, glasses, rings, gloves and helmets are the most popular wearable devices currently available on the market [1], which is projected to rise up to $\$ 30.2$ billion by 2018 [2]. According to Juniper Research, companies will spend up to $\$ 68.7$ million in wearable advertising by 2018 [3]. In genereal, wearable devices are defined as electronic technology to be incorporated in clothing or worn on the body, able to perform different tasks, such the tracking, monitoring of physiological functions and provide biofeedback [4].

The hardware of mobile devices improved considerably in recent years, but not the batteries, which take decades to double performance [5]. Consequently, energy is a very precious resource for mobile devices and wearables in particular. In addition, $75 \%$ of users consider battery lifetime as the main feature they look at while buying mobile devices [6].

Mobile Cloud Computing (MCC) augments performance of mobile devices by fostering task offloading [7]. The offloading can refer to both traffic offloading [8] or computation offloading. In the second case, offloaded tasks are executed remotely in the cloud to reduce processing and energy consumption of the mobile device. Offloading is a valid strategy not only for energy saving, but can also for expanding data storage and extending computing capacity [9], [10], [11], [12], [13]. Executing tasks on a system with higher computational power is especially useful for CPU-intensive applications [14], such as face recognition, a task which takes as long as 38 minutes if executed locally on smart glasses [15].

Offloading requires mobile devices to maintain data connection for communicating with remote servers, which is costly in terms of the consumed energy. To understand full benefit of computation offloading, the tradeoff between the increase in energy spent for communication and energy saved by avoiding local processing should be analyzed. A detailed analysis of cloud energy consumption has revealed that wireless access networks and not datacenters are the most energy hungry components of the cloud ecosystem [16], [17]. Computation offloading requires data to be first transmitted and processed before receiving back the results, which makes offloading time consuming.

In this paper, we analyze through analytical modeling the advantages brought by computation offloading to the cloud to wearable devices focusing on the energy and execution delay tradeoffs. Several works have already analyzed energy consumption and battery limitations of smartphones [18], [19], [20], [21] and wearable devices [15]. However, to the best of our knowledge, this is the first work providing comprehensive analysis of both computational and communication aspects of offloading. More precisely, the performed analysis considers energy and time needed to perform tasks. Understanding when and whether it is convenient to offload a task is crucial to extend battery lifetime and guarantee Quality of Service (QoS) to the users. For example, offloading parts of the delaysensitive application can save energy, but affect Quality of User Experience (QoUE).

The rest of the paper is structured as follows: Section II presents background on mobile cloud computing with an emphasis on wearable devices. Section III illustrates the model analyzing the cost of task offloading in terms of the energy consumed by devices and task completion time. Section IV provides performance evaluation and Section $\mathrm{V}$ concludes the work.

\section{Mobile Cloud Computing and Wearable DEVICES}

Fig. 1 illustrates a typical mobile cloud computing scenario. Wearable devices are often equipped with wireless $\mathrm{WiFi}$ and/or Bluetooth connectivity, but usually have no 3G/LTE interfaces, because of their significantly higher energy consumption [20], [21]. Nevertheless, as 3G/LTE connectivity is expected to be present in future devices, we include into our analysis the case when wearable devices exploit cellular connectivity using the user smartphone as relay. Furthermore, as smartphones can be used for offloading tasks in addition to clouds, it is important to take into account also their energy consumption.

For the aforementioned reasons, the proposed model accounts for all three main architectural components: wearable devices, smartphones, and cloud data centers. Computation 
Table i. Offloading scenarios. Values are Categorized as (H) High, (M) Medium, (L) Low and (-) No cost.

\begin{tabular}{|c|c|c|c|c|c|c|}
\hline \multirow{2}{*}{ OfFloAdING DEVICE } & \multirow{2}{*}{ SCENARIO } & \multirow{2}{*}{ LABEL } & \multicolumn{2}{|c|}{ COMPUTING } & \multicolumn{2}{|c|}{ COMMUNICATION } \\
\hline & & & ENERGY & TIME & ENERGY & TIME \\
\hline \multirow{4}{*}{ Wearable Device (w) } & Local processing (l) at the wearable device & $w-1$ & $\mathrm{H}$ & $\mathrm{H}$ & - & - \\
\hline & Offloading to smartphone (s) & $w-s$ & - & - & $\mathrm{L}$ & $\mathrm{L}$ \\
\hline & Offloading to cloud (c) via smartphone (s) & w-S-c & - & - & $\mathrm{L}$ & $\mathrm{H}$ \\
\hline & Offloading to cloud (c) via access point (ap) & w-ap-c & - & - & $\mathrm{L}$ & M \\
\hline \multirow{2}{*}{ Smartphone (s) } & Local processing (l) at the smartphone & s-1 & M & M & $\mathrm{L}$ & $\mathrm{H}$ \\
\hline & Offloading to cloud (c) & $\mathrm{s}-\mathrm{c}$ & - & - & $\mathrm{H}$ & $\mathrm{M}$ \\
\hline
\end{tabular}

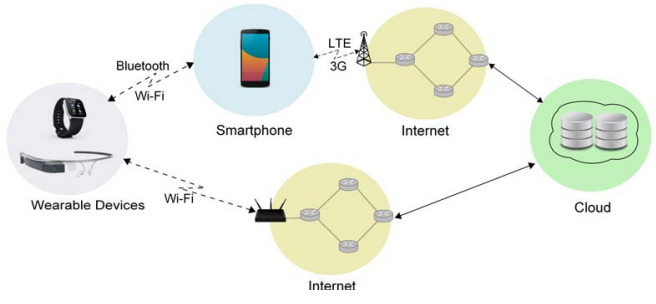

Fig. 1. Mobile cloud computing scenario for wearable devices

offloading can be performed in four different ways. In the first case, labeled in Table I as "w-l", wearable devices can execute complete tasks locally, which corresponds to no offloading. They use considerable amount of computing resources, drain energy, but can safe on communication with no delays introduced because of offloading. The second possibility is to offload processing from the wearable device to the smartphone (w-s). The offloaded task needs to be transmitted from wearable device to the smartphone. The smartphone performs processing and returns the result back, which introduces communication delays, but helps to avoid local processing at the wearable device. The third case, labeled as "w-s-c", allows the smartphone to relay and send the offloaded tasks to the cloud. Processing in the cloud is especially beneficial for computationally intensive tasks, but introduces higher communication delays to account for wide-area network delivery to distant servers. Finally, in the case labeled as "w-ap-c", wearable devices are connected to the cloud through WiFi. It involves only one radio link in the access and the processing is performed in the cloud.

From the smartphones' point of view, two scenarios can be identified. First, when they perform processing locally (s1), medium amount of energy is required with minimum cost of communications involved, due to the proximity to wearable device and low consumption of the WiFi/Bluetooth interfaces. On the other hand, the time necessary for computing and transmitting back to the wearable device results is high, as smartphones have limited computing power, which results in increased time of processing. Alternatively, the smartphone can offload tasks to the cloud (s-c). In such scenario, no computing is required at the smartphone, but communications require to keep alive both LTE and WiFi/Bluetooth interfaces, which inquires high energy consumption.

\section{COMPUting AND COMMUNICATION OFFLOADING MODEL}

Mobile devices can exploit different technologies for communications. Wearable devices are usually equipped with $\mathrm{WiFi}$, Bluetooth and Near Field Communication (NFC) interfaces or a combination of them. For the scope of this analysis, we
TABLE II. WiFi SETUP PARAMETERS

\begin{tabular}{ccl}
\hline SYMBOL & VALUE & DESCRIPTION \\
\hline$\rho_{i d}$ & $3.68 \mathrm{~W}$ & Power in idle mode \\
$\rho_{t x}$ & $0.37 \mathrm{~W}$ & Power during transmission \\
$\rho_{r x}$ & $0.31 \mathrm{~W}$ & Power during reception \\
$\lambda_{r}$ & $1000 \mathrm{fps}$ & Packet reception rate \\
$\lambda_{g}$ & $1000 \mathrm{fps}$ & Packet generation rate \\
$\gamma_{x r}$ & $0.09 \cdot 10^{-3} \mathrm{~J}$ & Processing energy during packet reception \\
$\gamma_{x g}$ & $0.11 \cdot 10^{-3} \mathrm{~J}$ & Processing energy during packet generation \\
\hline
\end{tabular}

consider WiFi technology, in particular the standard IEEE $802.11 \mathrm{~g}$. It provides high data rates (of up to $54 \mathrm{Mbit} / \mathrm{s}$ ) and the longest operating range for wearable devices. The most common technology for the communication between the smartphone and the cloud is cellular $3 \mathrm{G} / \mathrm{LTE}$. It supports data rates of up to $300 \mathrm{Mbit} / \mathrm{s}$ in the downlink and $75 \mathrm{Mbit} / \mathrm{s}$ in the uplink.

\section{A. Communications in Mobile Cloud Computing}

Mobile devices can exploit different technologies for communications. Wearable devices are usually equipped with $\mathrm{WiFi}$, Bluetooth and Near Field Communication (NFC) interfaces or a combination of them. For the scope of this analysis, we consider WiFi technology, in particular the standard IEEE $802.11 \mathrm{~g}$ as it provides high data rates (up to $54 \mathrm{Mbit} / \mathrm{s}$ ) and the longest operating range for wearable devices. The most common technology for the communication between the smartphone and the cloud is cellular $3 \mathrm{G} / \mathrm{LTE}$. It supports data rates of up to $300 \mathrm{Mbit} / \mathrm{s}$ in the downlink and $75 \mathrm{Mbit} / \mathrm{s}$ in the uplink.

Equation (1) describes the WiFi transmission time $T_{w}$ of $N$ packets. $T_{p}$ represents individual packet transmission time. $T_{a c k}$ is the time required for acknowledgment. DIFS and $S I F S$ are inter-frames spaces specified by the IEEE 802.11 standard. $B$ is a backoff time, which helps to avoid network contention if multiple nodes need to access the shared channel simultaneously.

$$
T_{w}=D I F S+B+N \cdot\left(T_{p}+T_{a c k}+S I F S\right) .
$$

To model WiFi power consumption $P_{w}$, we rely on the model proposed by Garcia et al. in [22]:

$$
P_{w}=\rho_{i d}+\rho_{t x} \cdot \tau_{t x}+\rho_{r x} \cdot \tau_{r x}+\gamma_{x g} \cdot \lambda_{g+} \gamma_{x r} \cdot \lambda_{r},
$$

where $\tau_{t x}$ and $\tau_{r x}$ are channel airtime percentage for transmission and reception respectively. Table II describes remaining parameters and their corresponding values used to validate the model.

The time $T_{l}$ spent for transmission of $D$ bytes sent at rate $r$ on LTE link is as follows:

$$
T_{l}=T_{p r}+(D \cdot 8) / r .
$$


$T_{p r}$ is the promotion time, which is the time necessary to allocate resources to the device. This involves switching from a low-power state to a high-power state for transmission. Equation (3) is applicable for both uplink and downlink traffic. According to the model presented in [23], the power consumption $P_{l}$ over the LTE link can be described as follows:

$$
P_{l}=\alpha_{u} \cdot t_{u}+\alpha_{d} \cdot t_{d}+\beta \text {. }
$$

Table III lists typical values for power consumption on LTE links.

\section{B. Computation Offloading from Wearable Devices}

Four different task execution models are available for wearable devices. They can execute tasks locally (w-1), offload to the smartphone (w-s), offload to the cloud (c) via smartphone (w-s-c) or offload to the cloud via WiFi access point (w-ap-c). The offloaded tasks are considered to be always accepted and processed in the cloud.

Local Processing in Wearable Devices (w-l): The simplest way is to start task execution locally. Task completion time is deterministic and depends on the computing power of the local hardware, which is quite limited for the majority of wearable devices. As a result, only tasks requiring low- and medium-size computing can be executed locally. While high-performance computing tasks would either take too long to complete or will drain the available battery power too fast.

Offloading from Wearable Devices to Smartphone (w-s): The nearest place to offload execution is a smartphone. Task execution can take benefits of larger computing, battery power, and storage resources of the smartphone, but require sending tasks for execution and receiving back the completion result. Yet, this communication occurs over short range and is energy efficient. The time needed to obtain results $T_{\mathrm{w}-\mathrm{s}}$ consists of the time necessary for sending data to the smartphone and the time to receive the results back using $\mathrm{WiFi}$ connection $T_{w}$ and the processing time $T_{s p}$ taken by the smartphone:

$$
T_{\mathrm{w}-\mathrm{s}}=T_{w}+T_{s p} \text {. }
$$

From the wearable device point-of-view, energy $E_{\mathrm{w}-\mathrm{s}}$ is only spent for communication purposes and can be described as follows:

$$
E_{\mathrm{w}-\mathrm{s}}=T_{w} \cdot P_{w} .
$$

We recall that definition of $P_{w}$ can be found in (2).

Offloading from Wearable Devices to Cloud via Smartphone (w-s-c): When the smartphone acts as a relay in offloading tasks to the cloud, the time needed to obtain the results $T_{\mathrm{w}-\mathrm{s}-\mathrm{c}}$ is defined as follows:

$$
T_{\mathrm{w}-\mathrm{s}-\mathrm{c}}=T_{w}+T_{l}+\delta_{i}+T_{c p},
$$

where $T_{w}$ is the WiFi communication time between wearable device and the smartphone, $T_{l}$ corresponds to the time spent over the LTE link as per (3) and $\delta_{i}$ corresponds to the wide-area network delay [24], while $T_{c p}$ corresponds to the time taken by the cloud to perform computing. Energy consumption remains identical to the case (w-s) and it is described in (6).

Offloading from Wearable Devices to Cloud via Access Point (w-ap-c): Similarly to the second and third cases (w$\mathrm{s}, \mathrm{W}-\mathrm{S}-\mathrm{c})$, when wearable devices offload tasks to the remote cloud without using a smartphone as a relay, wearables spend
TABLE III. LTE SETUP PARAMETERS

\begin{tabular}{ccl}
\hline SYMBOL & VALUE & DESCRIPTION \\
\hline$\alpha_{u}$ & $438.39 \cdot 10^{-9} \mathrm{~W} / \mathrm{bps}$ & Power for bps in uplink \\
$\alpha_{d}$ & $51.97 \cdot 10^{-9} \mathrm{~W} / \mathrm{bps}$ & Power for bps in downlink \\
$\alpha_{p}$ & $1210.7 \cdot 10^{-3} \mathrm{~W} / \mathrm{bps}$ & Power for promotion \\
$\beta$ & $1288.04 \cdot 10^{-9} \mathrm{~W}$ & Idle Power \\
\hline
\end{tabular}

energy only to communicate with the AP (6). To be generic, the proposed model differentiates between distances of wearable devices and the AP and distance between wearable devices and the smartphone. This allows capturing different channel conditions and accounts to variable latencies. In this scenario, the time wearable devices need to receive back the results is described as follows:

$$
T_{\mathrm{w}-\mathrm{ap}-\mathrm{c}}=T_{w}+\delta_{i}+T_{c p},
$$

where $T_{w}+\delta_{i}$ corresponds to the communications with the cloud through the AP and the Internet and $T_{c p}$ is the processing time in the cloud.

Fig. 2 shows the time and energy comparison between the different cases having considered a face recognition task with images of variable size. Face recognition is both computingand communication-intensive task [25]. We used Matlab model to derive relationship between the size of the picture and the number of instructions. The power spent for processing is set to be equal to $3318 \mathrm{~mW}$ per instruction on the wearable device and $2845 \mathrm{~mW}$ on the smartphone [15], [20]. Tables II and III list the communication parameters used for analytical validation of the model. As expected, Fig. 2 shows that offloading appears to be highly beneficial in terms of responsiveness and energy consumption as the amount of data to be transferred and processed increases. Specifically, wearable devices have advantage to offload tasks for local execution in the smartphone over offloading to the cloud when the data size is smaller than $1 \mathrm{MB}$. For objects of a larger size, the cloud always provides faster responses. In addition, Fig. 2(a) highlights that WiFi technology should be preferred over LTE when tasks are executed in the cloud.

\section{Computation Offloading from Smartphones}

From the smartphones' point of view, upon receiving a task from wearable devices they can either (a) execute the task locally (s-1) or (b) offload the task to the cloud (s-c).

Local Processing in Smartphones (s-l): In such a scenario, the smartphone receives data from the wearable device performs the task locally and sends the results back. Communications happen through the WiFi link only. As a result, we define the time $T_{\mathrm{s}-1}$ spent by the smartphone in assisting the wearable device for offloading as:

$$
T_{\mathrm{s}-1}=T_{w}+T_{s p}
$$

where $T_{w}$ corresponds to the communication time through $\mathrm{WiFi}$ and $T_{s p}$ the time spent for local processing. Smartphones spend energy $E_{\mathrm{s}-1}$ for both computing and communication:

$$
E_{\mathrm{s}-1}=T_{w}+P_{w}+T_{s p} \cdot P_{s p},
$$

where $P_{w}$ corresponds to the $\mathrm{WiFi}$ power consumption as per (2) and $P_{s p}$ is the power spent for local processing.

Offloading from Smartphones to Cloud (s-c): Smartphones can receive data from wearable devices and to perform offloading to the cloud. In such case, no energy costs are associated 


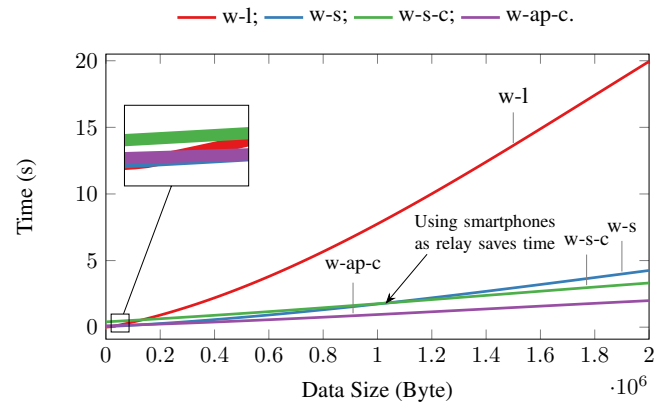

(a)

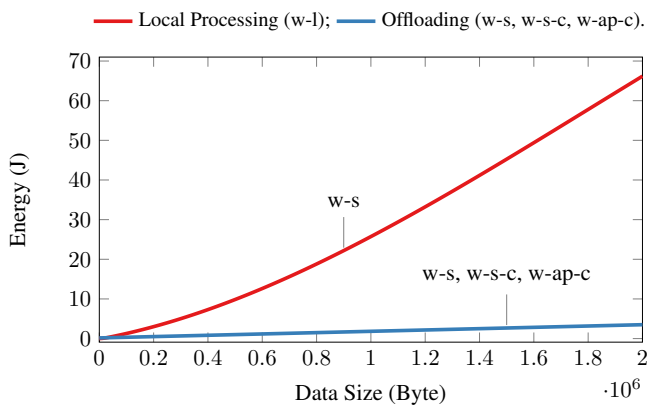

(b)

Fig. 2. Wearable device (a) processing and communication time and (b) energy consumption

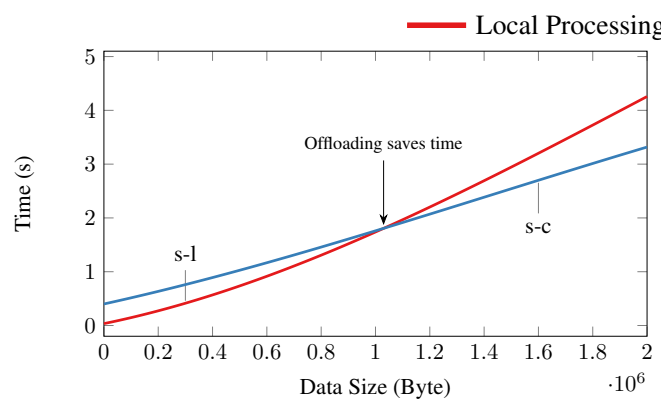

(a)

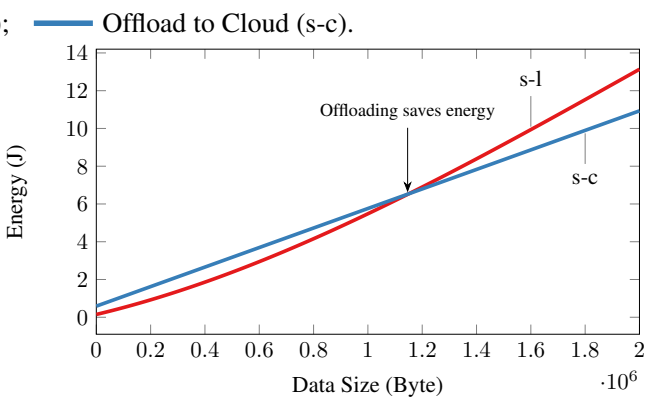

(b)

Fig. 3. Smartphone (a) processing and communication time and (b) energy consumption

with processing, but the smartphone is required to keep active both WiFi and LTE interfaces for communication for the time $T_{w}$ and $T_{l}$. As a result, $T_{\mathrm{s}-\mathrm{c}}$ the time spent by the smartphone in assisting the wearable device for offloading is defined as:

$$
T_{\mathrm{s}-\mathrm{c}}=T_{w}+T_{l} \text {. }
$$

The energy spent by the smartphone is defined as:

$$
E_{\mathrm{s}-\mathrm{c}}=T_{w} \cdot P_{w}+T_{l} \cdot P_{l}+\alpha_{p} \cdot T_{p r},
$$

where where $P_{w}$ and $P_{l}$ correspond to the WiFi and LTE power consumption defined in (2) and (4) respectively, while $\alpha_{p}$ corresponds to the power spent by smartphones during promotion time $T_{p r}$ as per Table III.

Fig. 3 shows the time and energy for smartphones. In contrast with the results obtained for wearables, Fig. 3(b) shows that it becomes more energy efficient if smartphones perform offloading if the size of data transfer is larger than 1.2 MB. This value is in full agreement with the expected during analysis value.

\section{PERformance EVAluation}

This section provides performance evaluation performed with NS-3 network simulator extended with LTE functionality from LENA project framework. Similarly to Section III, face recognition is the application used for our analysis.

\section{A. System Scenario}

Google Glasses have been used as wearable device. They are equipped with a dual core ARM Cortex-A9 CPU with maximum frequency at $1 \mathrm{GHz}$ [26]. This architecture is able to offer a maximum computational power of 5000 DMIPS. The power consumed to perform an OpenCV detection algorithm is $3318 \mathrm{~mW}$, while data transmission consumes $653 \mathrm{~mW}$ using WiFi at $734 \mathrm{Kbps}$ [15], [27]. LG Nexus 5 is the smartphone used for simulations. It is equipped with a quad core Qualcomm Snapdragon with a maximum computational power of 30645 DMIPS [28]. The simulated cloud is assumed to have a computational power of an Intel Core i7 $3770 \mathrm{~K}$ able to elaborate a maximum of 106926 DMIPS at $3.9 \mathrm{GHz}$ [29].

For communications, the simulated $\mathrm{WiFi}$ data rates range from $734 \mathrm{Kbps}$ to a maximum of $24 \mathrm{Mbps}$ [15]. For LTE, in the uplink the supported data rates range from $0.924 \mathrm{Mbps}$ up to $20 \mathrm{Mbps}$ while in downlink the considered range is 2.24-40.2 Mbps. Distances between the devices (wearable and smartphones) and antennas (WiFi AP and LTE antennas) range between 0.5 and $15 \mathrm{~m}$ for WiFi and from 50 to $500 \mathrm{~m}$ for LTE. The size of simulated graphical objects range from $50 \mathrm{~KB}$ to a maximum size of $2 \mathrm{MB}$.

For analysis of energy consumption, the setting used for computing is exactly the same of Section III. Wearable devices spend $653 \mathrm{~mW}$ and smartphone $1749 \mathrm{~mW}$ for communications over the WiFi links [15] and smartphones consume 2200 $\mathrm{mW}$ [21] over the LTE links.

\section{B. Results}

Fig. 4 shows the results of the simulation from the point of view of the wearable device. Similar to the model, the decision whether to offload a task or to execute locally depends on the amount of the data to be processed. For small data sizes, below $150 \mathrm{~KB}$, the difference in execution time is marginal, while for bigger amounts of data to be processed the results are consistent with the proposed model. Offloading results being always more energy efficient as compared to local processing.

Fig. 5 shows the simulation results for smartphones. With respect to previous analysis, the behaviour of the charts is 


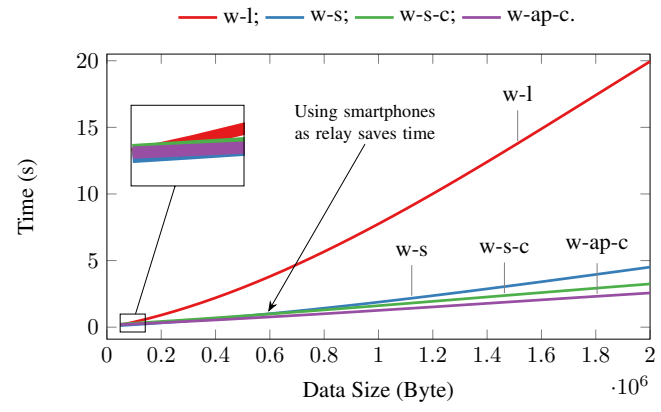

(a)

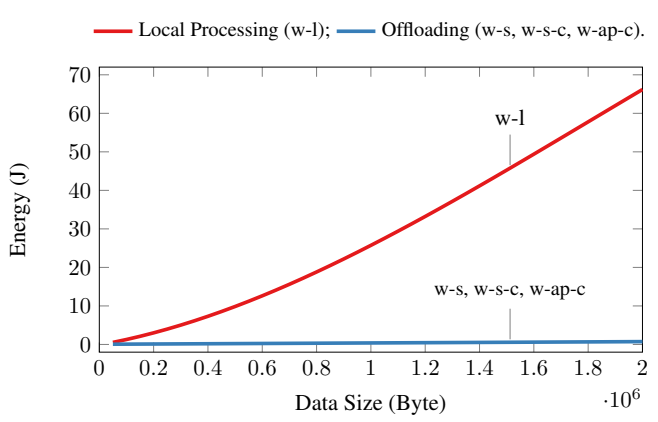

(b)

Fig. 4. Wearable device (a) processing and communication time and (b) energy consumption

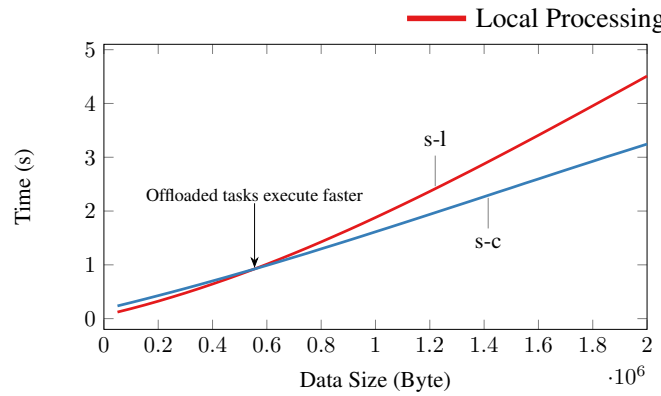

(a)

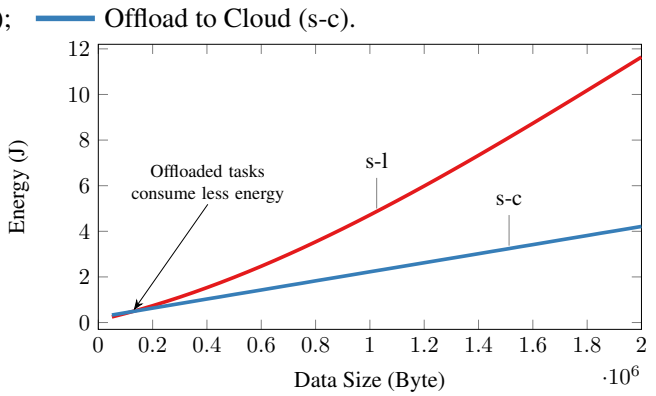

(b)

Fig. 5. Smartphone (a) processing and communication time and (b) energy consumption

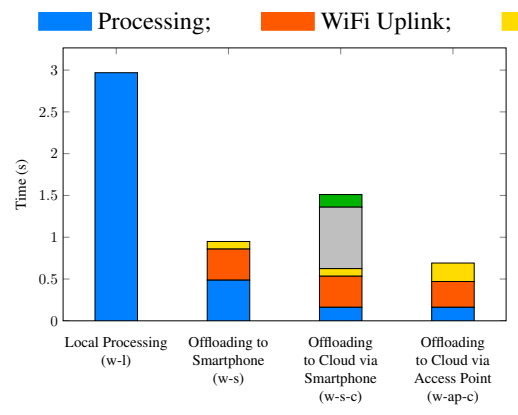

(a)

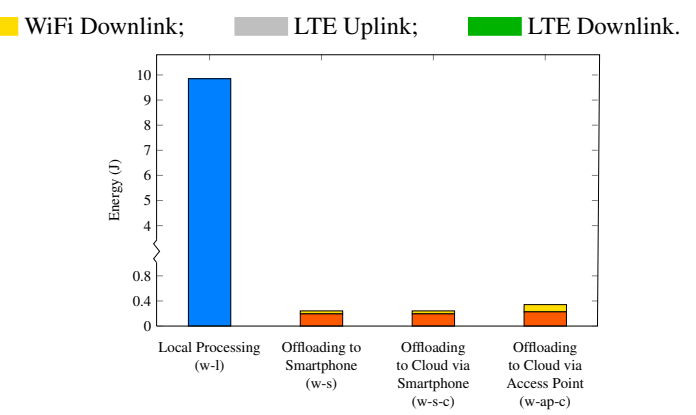

(b)

Fig. 6. Distribution of (a) processing and communication time and (b) energy consumption for wearable devices

consistent with the model only for time responsiveness. More precisely, performing offloading starts being beneficial for amounts of data to be processed larger than $600 \mathrm{~KB}$. For energy consumption, instead, offloading is practically always beneficial as the threshold is around $150 \mathrm{~KB}$. The results presented in Fig. 5 differ from the theoretical model. This is especially evident for energy consumption (see comparison of Fig. 5(b) and Fig. 3(b)). On one hand, it is because NS3 implements more realistic models for communication, while on the other hand the results in Fig. 5 are averages having considered different channel conditions, which can not be captured by the theoretical model.

Fig. 6 shows task execution time and energy consumption measured at the wearable device during offloading of a $500 \mathrm{~KB}$ file. If performed locally, task execution results being a heavy operation. Indeed, offloading is beneficial for the lifetime of the device as all cases outperform local execution in terms of both time and energy. From time perspective, the best way is to offload tasks to the cloud using WiFi connection, because cloud performs computing faster than a mobile device. Direct connection with the cloud avoids communication over the timeconsuming LTE network at the expense in energy consumption. Because of the need to cross the Evolved Packet Core (EPC) network [30], communications over LTE are time consuming and it becomes clear comparing the cases (w-s) and (w-s-c) in Fig. 6(a). For this amount of data $(500 \mathrm{~KB})$, the local processing at the smartphone leads to a faster response to the wearable device with the same energy cost. This can be considered as an intermediate case, as the data size is not small enough to be efficiently processed on the wearable device and not so large to be sent to the cloud.

Fig. 7 shows task execution time and energy consumption measured at the smartphone during offloading of a picture of $500 \mathrm{~KB}$. Local processing at the smartphone provides faster response if compared to offloading the task to the cloud because of the communication over the LTE network. Indeed, even if the cloud can perform the task faster, the time spent in communication over LTE will affect negatively the 


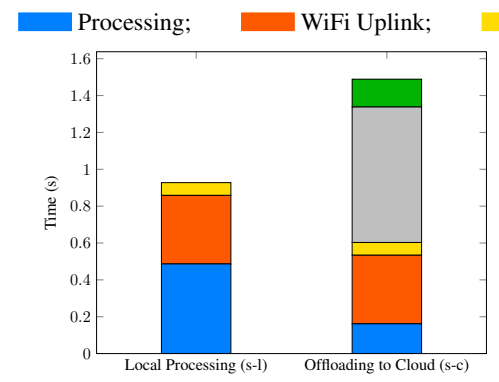

(a)

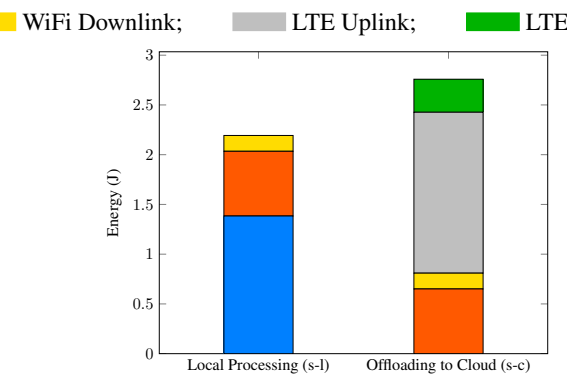

(b)

Fig. 7. Distribution of (a) processing and communication time and (b) energy consumption for smartphones

performance with respect of both time and energy.

\section{CONClusion}

In this paper, we investigate task offloading performed by wearable devices in mobile cloud computing. Multiple offloading scenarios are taken into account, including local processing (at wearables and at smartphones) and according to the technology used (LTE and WiFi). The energy spent for computing and communications and the time needed to transmit data and obtain the results back are the main performance metrics analyzed, measured from the perspective of both the smartphones and wearable devices.

The proposed model shows that there is no a single optimum way for task execution. It can be optimized by execution time or the energy consumption. However, knowing in advance the best way to perform a task affects positively the mobile devices acting in the scenario. Above all, this is especially important for the case of wearables with limited battery lifetime.

Future work will be devoted to extend the proposed model to other technologies than WiFi and LTE and perform measurements with real wearable devices.

\section{ACKNOWLEDGMENT}

The authors would like to acknowledge the funding from National Research Fund, Luxembourg in the framework of ECO-CLOUD and iShOP projects.

\section{REFERENCES}

[1] Wearable. (2014) 50 Wearable gamechangers for 2015. [Online].

[2] BBC Research. (2014, Feb.) Wearable computing: Technologies, applications and global markets. [Online].

[3] Juniper Research, "Digital advertising: Online, mobile \& wearables 2015-2019," 2015.

[4] K. Tehrani and M. Andrew. (2014, Mar.) Wearable technology and wearable devices: Everything you need to know. [Online]. Available: http://www.wearabledevices.com/what-is-a-wearable-device

[5] F. Schlachter, "No Moore's law for batteries," Proceedings of the National Academy of Sciences, vol. 110, no. 14, p. 5273, 2013.

[6] K. Kumar, J. Liu, Y.-H. Lu, and B. Bhargava, "A survey of computation offloading for mobile systems," Mobile Network Applications, vol. 18, no. 1, pp. 129-140, Feb. 2013.

[7] N. Fernando, S. W. Loke, and W. Rahayu, "Mobile cloud computing: A survey," Future Generation Computer Systems, vol. 29, no. 1, pp. 84 - 106, 2013.

[8] C. Fiandrino, D. Kliazovich, P. Bouvry, and A. Zomaya, "Networkassisted offloading for mobile cloud applications," in IEEE ICC, London, United Kingdom, Jun. 2015.

[9] M. Segata, B. Bloessl, C. Sommer, and F. Dressler, "Towards energy efficient smart phone applications: Energy models for offloading tasks into the cloud," in IEEE ICC, June 2014, pp. 2394-2399.
[10] L. Xiang, S. Ye, Y. Feng, B. Li, and B. Li, "Ready, set, go: Coalesced offloading from mobile devices to the cloud," in INFOCOM'14, 2014, pp. 2373-2381.

[11] $\mathrm{H}$. Wu and K. Wolter, "Tradeoff analysis for mobile cloud offloading based on an additive energy-performance metric," in 8th International Conference VALUETOOLS, Feb 2015.

[12] M. Barbera, S. Kosta, A. Mei, and J. Stefa, "To offload or not to offload? The bandwidth and energy costs of mobile cloud computing," in Proceedings IEEE INFOCOM, April 2013, pp. 1285-1293.

[13] K. Kumar and Y.-H. Lu, "Cloud computing for mobile users: Can offloading computation save energy?" Computer, vol. 43, no. 4, pp. 51-56, Apr. 2010.

[14] The Bluetooth Special Interest Group (SIG) Inc. (2010, Jun.) Core version 4.0.

[15] R. LiKamWa, Z. Wang, A. Carroll, F. X. Lin, and L. Zhong, "Draining our glass: An energy and heat characterization of Google Glass," CoRR, vol. abs/1404.1320, 2014.

[16] CEET, "The power of wireless cloud," 2013, White Paper.

[17] C. Fiandrino, D. Kliazovich, P. Bouvry, and A. Zomaya, "Performance and energy efficiency metrics for communication systems of cloud computing data centers," IEEE Transactions on Cloud Computing, 2015.

[18] G. P. Perrucci, F. H. P. Fitzek, and J. Widmer, "Survey on energy consumption entities on the smartphone platform," in VTC Spring'11, 2011, pp. 1-6.

[19] L. Ardito, G. Procaccianti, M. Torchiano, and G. Migliore, "Profiling power consumption on mobile devices," in ENERGY: The Third International Conference on Smart Grids, 2013, pp. 102-106.

[20] A. Carroll and G. Heiser, "The systems hacker's guide to the galaxy: Energy usage in a modern smartphone," in Asia-Pacific Workshop on Systems (APSys). Singapore: ACM, July 2013, p. 7.

[21] M. Y. Malik, "Power consumption analysis of a modern smartphone," CoRR, vol. abs/1212.1896, 2012.

[22] A. Garcia-Saavedra, P. Serrano, A. Banchs, and G. Bianchi, "Energy consumption anatomy of 802.11 devices and its implication on modeling and design," in Proceedings of the 8th International Conference on Emerging Networking Experiments and Technologies, ser. CoNEXT ' 12. New York, NY, USA: ACM, 2012, pp. 169-180.

[23] J. Huang, F. Qian, A. Gerber, Z. M. Mao, S. Sen, and O. Spatscheck, "A close examination of performance and power characteristics of $4 \mathrm{G}$ LTE networks," in MobiSys. ACM, 2012, pp. 225-238.

[24] Verizon Enterprise Solutions. (2015) IP latency statistics.

[25] T. Soyata, R. Muraleedharan, C. Funai, M. Kwon, and W. Heinzelman, "Cloud-vision: Real-time face recognition using a mobile-cloudlet-cloud acceleration architecture," in IEEE ISCC, July 2012, pp. 59-66.

[26] ARM Ltd. (2014) Cortex-a9 processor. [Online]. Available: http: //www.arm.com/cortex-a9.php

[27] Google. (2014) Google Glass. [Online]. Available: https://www.google. com/glass/start/

[28] LG Corporation. (2014) LG NEXUS 5 - Technical Specifications. [Online]. Available: http://www.lg.com/uk/mobile-phones/lg-D821/ technical-specifications

[29] Intel Corporation. (2012) Intel $®$ Core $^{\mathrm{TM}} \mathrm{i} 7-3770 \mathrm{~K}$ Processor. [Online]. Available: http://ark.intel.com/products/65523

[30] C. Fiandrino, D. Kliazovich, P. Bouvry, and A. Zomaya, "NC-CELL: Network coding-based content distribution in cellular networks for cloud applications," in IEEE GLOBECOM, Dec 2014, pp. 1205-1210. 